\title{
A new approach in bowel preparation before colonoscopy in patients with constipation: A prospective, randomized, investigator-blinded trial
}

Murat Yıldar, İsmail Yaman, Murat Bașbuğ, Faruk Çavdar, Hasan Topfedaisi, Hayrullah Derici

ABSTRACT

Cite this paper as:

Yıldar M, Yaman I, Başbuğ

M, Çavdar D, Topfedaisi H,

Derici H. A new approach in

bowel preparation before

colonoscopy in patients with

constipation: A prospective,

randomized, investigator-

blinded trial. Turk J Surg

2017; 33(1): 29-32.

Department of General Surgery, Ballkesir University School of Medicine, Balıkesir, Turkey

Address for Correspondence Murat Yildar

e-mail: muratyildar@hotmail.com

Received: 04.05 .2015

Accepted: 06.09.2015

(c) Copyright 2017

by Turkish Surgical Association

Available online at

www.turkjsurg.com
Objective: Enema administration in the morning of routine colonoscopy is known to be useless. However, the potential bowel cleansing effects of distal colon emptying with enema prior to purgatives are not known. The aim of this study is to investigate the effects of enema use before purgatives in preparation for colonoscopy.

Material and Methods: Two hundred twenty-seven patients were randomly assigned into three groups; enema before purgative use, enema after purgative use, and no enema. Patients were compared in terms of age, sex, BMI, Rome III constipation criteria, history of abdominal surgery, tolerance to the preparation procedure, complications during preparation such as nausea, vomiting, headache and dizziness, cecal insertion time, total duration of colonoscopy, polyp determination rate and colonic cleansing based on the Boston Bowel Preparation Scale.

Results: One hundred two (44.9\%) patients were male and 125 (55.1\%) female. The mean age and BMI was $55.4 \pm 11.8$ years and $28.8 \pm 4.7$, respectively. No difference was observed between the groups in terms of sex, age, or BMI. The number of fulfilled Rome criteria and of previous abdominal surgeries were significantly higher in females than in men. Right colon Boston Bowel Preparation Scale score was higher in the group using enemas before purgatives than the scores of other groups. This improvement was statistically significant in the female patient group with higher constipation rate.

Conclusions: Use of enemas before purgatives in patients with constipation significantly improves adequacy of right colon cleansing.

Keywords: Bowel preparation, colonoscopy, constipation, sennoside $A \& B$, sodium phosphate enema

\section{INTRODUCTION}

Colonoscopy is widely used for the diagnosis and treatment of colon lesions. Adequate bowel cleansing forms the basis of successful colonoscopy (1). Purgatives are widely used for bowel cleansing (2). Experimental and clinical studies aimed at providing optimum colon cleansing are still being performed.

Solutions containing polyethylene glycol (PEG) and sodium phosphate (NaP) are generally used in colonoscopy preparations. The sennosides are generally used in combination with PEG. The use of sennosides without PEG combination is controversial (3). Enema is an agent that evacuates the distal colon and was a basic component of colonoscopy preparation before the introduction of PEG (2). However, it was later reported that additional enema use following colonic cleansing with purgatives was useless and caused patient discomfort (4). With this anecdotal information, the colonoscopy preparation document prepared by the American Society for Gastrointestinal Endoscopy (ASGE) recommended the use of enemas in individuals in whom poor preparation was observed during colonoscopy or in case of presence of de-functional bowel segment such as Hartmann's procedure (2). Despite these recommendations, enemas are being routinely used before colonoscopy as a standard approach in colon cleansing protocols in some general surgery and gastroenterological endoscopy units.

Sloots et al. (5) reported that bowel cleansing shortened colonic transit time, especially in patients with constipation. Bowel cleansing was performed with Klean-Prep ${ }^{\oplus}$ in both patients and volunteers in their study. They reported that radioactive markers were expelled more quickly from the colon with bowel cleansing. In light of these findings, we thought that emptying the distal colon before purgative use can enhance the effect of purgatives by increasing bowel activity. With this aim, we investigated the effects of enema administration before purgative use on colonoscopy preparation.

\section{MATERIAL AND METHODS}

This prospective study was performed on patients who were referred to our clinic for elective total colonoscopy either for screening or evaluation of abdominal pain or fecal occult blood positivity. Patients younger than 18 years of age or with previous colorectal resection were excluded. All colonoscopies were performed by experienced endoscopists performing more than 150 colonoscopies annually, between 9:00 AM and 2:00 PM. A video colonoscope (EC-380LKp; Pentax, Japan) was used. Midazolam + pentidine HCL was used for sedation in all procedures. Patients were monitored during colonoscopy and their blood pressure, heart rate and pe- 
ripheral oxygen saturation were kept under control. Midazolam + pentidine $\mathrm{HCL}$ was administered by a nurse under endoscopist supervision. The standard oral purgative agent used in the pre-colonoscopy cleansing protocol contained sennoside $A+B$ calcium (XM ${ }^{\oplus}$; solution $250 \mathrm{~mL}$, Yenişehir Lab., Ankara, Turkey). The enema administered by the rectal route contained sodium hydrogen phosphate and disodium hydrogen phosphate $\left(\mathrm{BT}^{\oplus}\right.$ enema 210 mL, Yenişehir Lab., Ankara, Turkey). Approval for this prospective observational study was obtained from Çanakkale Onsekiz Mart University Clinical Research Ethical Committee. All participants were informed of potential complications before the procedure, and written informed consent was obtained.

Patients were randomly assigned into one of three groups using sequential group forms by endoscopy nurses. Patients in all groups were given a clear diet without pulp one day before the procedure. Purgatives were given twice, at 11:00 AM and 6:00 $\mathrm{PM}$, at a rate of $125 \mathrm{~mL}$, on the day before colonoscopy. Group 1 (Pre-enema) patients were administered fleet enema by the rectal route at 10:00 AM before purgative administration, one day before the procedure. Group 2 (Post-enema) patients received enema by the rectal route in the hospital on the day of colonoscopy. Group 3 (No enema) patients did not receive enema.

Patients were assessed in terms of constipation using the Rome constipation criteria and their demographic data were recorded before colonoscopy (6). Previous abdominal surgeries were noted. Preparatory procedure tolerance was defined as very comfortable, comfortable, uncomfortable and very uncomfortable, and symptoms such as nausea, vomiting, abdominal pain, dizziness and headache were described as none, mild, moderate or severe. Colonic cleansing was scored by the endoscopist blind to the cleansing protocol with the Boston Bowel Preparation scale (BBPS) (Table 1) (7). The endoscopist scored the right colon (the cecum and ascending colon), transverse colon (hepatic and splenic flexures), and the left colon (descending colon, sigmoid colon and rectum) separately. The minimum total score was 0 and maximum total score was 9. Cecal intubation and total colonoscopy times and presence of polyp or tumor were also recorded.

\section{Statistical Analysis}

Data were summarized as means, standard deviation, median (min-max) and percentages. ANOVA or the Kruskal-Wallis test were used for intergroup comparisons depending on normal distribution of data (using the Lilliefors test), with the Post Hoc test if necessary. Categorical data were compared using the chi square test. Values less than 0.05 were regarded as statistically significant. Analysis was performed with Statistical Package for the Social Sciences 20 software (SPSS Inc.; IBM, Armonk, NY, USA).

\section{RESULTS}

\section{Patient Characteristics}

Patients identified as not adhering to the diet or with incomplete colonoscopy due to pain were excluded from the study. Of the remaining 227 patients, 102 (44.9\%) were male and 125 (55.1\%) female. The mean age and BMI were $55.4 \pm 11.8$ and 28.8 \pm 4.7 , respectively. The groups were similar in terms of age, sex or BM (Table 2). The mean number of fulfilled Rome constipation criteria were higher in female patients than in males $(1.3 \pm 1.8$ and $0.8 \pm 1.4$, $\mathrm{p}=0.4$ ). There was no statistically significant difference between the groups in terms of Rome criteria (Table 2). Evaluation of pre-
Table 1. Boston Bowel Preparation Scale

0 Unprepared colon segment with mucosa not seen due to solid stool that cannot be cleared

1 Portion of mucosa of the colon segment seen, but other areas of the colon segment not well seen due to staining, residual stool and/or opaque liquid

2 Minor amount of residual staining, small fragments of stool and/ or opaque liquid, but mucosa of colon segment seen well

3 Entire mucosa of colon segment seen well with no residual staining, small fragments of stool or opaque liquid

Table 2. All groups' demographic data. Lengths of procedure and polyp detection rates

\begin{tabular}{|c|c|c|c|c|}
\hline & Pre-enema & Post-enema & No enema & p \\
\hline Number (No.) & 78 & 78 & 71 & \\
\hline Age $^{*}$ & $55.1 \pm 12.5$ & $55.6 \pm 11.9$ & $55.6 \pm 11.1$ & 0.958 \\
\hline \multicolumn{5}{|l|}{ Sex } \\
\hline Female $^{\#}$ & $42(53.8)$ & $44(56.4)$ & $39(54.9)$ & \multirow[t]{2}{*}{0.949} \\
\hline Male $^{\#}$ & $36(46.2)$ & $34(43.6)$ & $32(45.1)$ & \\
\hline Body mass index* & $28.7 \pm 4.6$ & $29.3 \pm 5.0$ & $28.4 \pm 4.3$ & 0.498 \\
\hline Rome criteria* & $1.0 \pm 1.5$ & $1.1 \pm 1.8$ & $1.1 \pm 1.7$ & 0.532 \\
\hline Cecalentubation time* & * $9.8 \pm 5.4$ & $8.8 \pm 4.3$ & $9.0 \pm 4.0$ & 0.361 \\
\hline Length of procedure* & $17.6 \pm 7.2$ & $16.5 \pm 5.4$ & $17.2 \pm 7.3$ & 0.637 \\
\hline Polyp detection rate ${ }^{*}$ & $26(33.3)$ & $21(26.9)$ & $20(28.2)$ & 0.670 \\
\hline
\end{tabular}

Datas are presented as *mean \pm standard deviation, "n (\%).

dominal surgery in 22/125 (17.6\%) women and in 12/102 (11.7\%) men, and conventional open abdominal surgery in $28 / 125$ (22.4\%) women and in 5/102 (4.9\%) men. Female patients had a significantly higher number of previous surgeries $(p<0.001)$.

\section{Patient Tolerance and Side-Effects}

Patient satisfaction with the preparation procedure was $86.4 \%$ (196/227). No significant difference was determined in preparation procedure tolerance in terms of complications such as nausea, vomiting, abdominal pain, dizziness and headache (Table 3).

\section{Effectiveness of Colonic Cleansing}

There was no significant difference between the groups in terms of total BBPS scores $(p=0.469)$. Right colon BBPS scores was increased with pre-purgative enema use, but the increase was not significant as compared to other groups $(p=0.109)$. Comparison between women only, excluding men, revealed a significantly higher right colonic cleansing score in the group using enemas before purgatives as compared to other groups. No difference was determined between the groups in terms of the other parameters investigated. The effect on the study groups' BBPS scores in male and female patients is shown in Table 4.

\section{Duration of Colonoscopy and Other Findings}

Mean cecal intubation time was $9.2 \pm 4.6 \mathrm{~min}$, and total duration of colonoscopy was $17 \pm 6.7 \mathrm{~min}$. Cecal intubation and total colonoscopy times were similar in all three groups (Table 2). One or more polyps were detected in 67 (29.5\%), and tumoral lesions were detected in 11 (4.8\%) patients. The rates of poyp detection were also similar in all three groups (Table 2). 
Table 3. Tolerance to preparation procedure in all groups, nausea, vomiting, abdominal pain, dizziness and headache

\begin{tabular}{|lcccc}
\hline & $\begin{array}{c}\text { Pre-enema } \\
(\mathbf{n}=78)\end{array}$ & $\begin{array}{c}\text { Post-enema } \\
(\mathbf{n}=78)\end{array}$ & $\begin{array}{c}\text { No enema } \\
(\mathbf{n}=71)\end{array}$ & $\mathbf{p}^{\dagger}$ \\
\hline Very comfortable & $39(50.0)$ & $44(56.4)$ & $27(38.6)$ & 0.336 \\
\hline Comfortable & $29(37.2)$ & $25(32.1)$ & $32(45.7)$ \\
\hline Uncomfortable & $9(11.5)$ & $9(11.5)$ & $11(15.7)$ \\
\hline Very uncomfortable & $1(1.3)$ & $0(0)$ & $0(0)$ \\
\hline & & \multicolumn{2}{c}{ Nausea } \\
\hline None & $57(73.1)$ & $54(69.2)$ & $47(66.2)$ & 0.349 \\
\hline Mild & $15(19.2)$ & $22(28.2)$ & $17(23.9)$ \\
\hline Moderate & $5(6.4)$ & $2(2.6)$ & $7(9.9)$ \\
\hline Severe & $1(1.3)$ & $0(0)$ & $0(0)$ \\
\hline Shi to preparation procedure
\end{tabular}

\section{DISCUSSION}

Evacuation of the distal colon with enemas immediately before purgative use in individuals undergoing preparation for colonoscopy significantly improved right colonic cleansing in this study, particularly in women. It has been reported that fecal impaction in the rectum has an inhibitory effect on bowel movements (5). We think that the probable reason why enema increased right colonic cleansing in this study is that it potentializes the purgative effect by emptying the rectum prior to purgative use. This observation in the female patient group was attributed to the higher prevalence of constipation in females than in males (8).
Table 4. Cleansing scores for colon segments according to the BBPS scale for men and women in all groups

\begin{tabular}{|c|c|c|c|c|c|c|}
\hline \multirow[b]{2}{*}{$\begin{array}{l}\text { Location } \\
\text { and score }\end{array}$} & \multicolumn{3}{|c|}{ Female } & \multicolumn{3}{|c|}{ Men } \\
\hline & $\begin{array}{c}\text { Pre- } \\
\text { enema } \\
(n=42)\end{array}$ & $\begin{array}{c}\text { Post- } \\
\text { enema } \\
(n=44)\end{array}$ & $\begin{array}{c}\text { No } \\
\text { enema } \\
(\mathrm{n}=39)\end{array}$ & $\begin{array}{c}\text { Pre- } \\
\text { enema } \\
(n=36)\end{array}$ & $\begin{array}{c}\text { Post- } \\
\text { enema } \\
(n=34)\end{array}$ & $\begin{array}{c}\text { No } \\
\text { enema } \\
(n=32)\end{array}$ \\
\hline \multicolumn{7}{|l|}{ Right colon $^{\dagger}$} \\
\hline 3 & $18(42.9)$ & $11(25)$ & $9(23.1)$ & $11(30.6)$ & $12(35.3)$ & $12(37.5)$ \\
\hline 2 & $19(45.2)$ & $17(38.6)$ & $18(46.2)$ & $20(55.6)$ & $16(47.1)$ & $13(40.6)$ \\
\hline 1 & $5(11.9)$ & $15(34.1)$ & $12(30.8)$ & $4(11.1)$ & $5(14.7)$ & $6(18.8)$ \\
\hline 0 & $0(0)$ & $1(2.3)$ & $0(0)$ & $1(2.8)$ & $1(2.9)$ & $1(3.1)$ \\
\hline$p^{t+}$ & & 0.017 & & & 0.993 & \\
\hline \multicolumn{7}{|c|}{ Transverse colon ${ }^{\dagger}$} \\
\hline 3 & $27(64.3)$ & $22(50)$ & $26(66.7)$ & $21(58.3)$ & $18(52.9)$ & $24(75)$ \\
\hline 2 & $13(31.0)$ & $16(34.4)$ & $8(20.5)$ & $9(25)$ & $14(41.2)$ & $7(21.9)$ \\
\hline 1 & $2(4.8)$ & $6(13.6)$ & $5(12.8)$ & $6(16.7)$ & $1(2.9)$ & $1(3.1)$ \\
\hline 0 & $0(0)$ & $0(0)$ & $0(0)$ & $0(0)$ & $1(2.9)$ & $0(0)$ \\
\hline $\mathrm{p}^{+t}$ & & 0.245 & & & 0.147 & \\
\hline \multicolumn{7}{|l|}{ Left colon ${ }^{+}$} \\
\hline 3 & $20(47.6)$ & $27(61.4)$ & $25(64.1)$ & $20(58.3)$ & $20(58.86)$ & $18(56.3)$ \\
\hline 2 & $19(45.2)$ & $11(25)$ & $10(25.6)$ & $11(30.6)$ & $13(38.2)$ & $10(31.3)$ \\
\hline 1 & $3(7.1)$ & $6(13.6)$ & $4(10.3)$ & $4(11.1)$ & $0(0)$ & $4(12.5)$ \\
\hline 0 & $0(0)$ & $0(0)$ & $0(0)$ & $0(0)$ & $1(2.9)$ & $0(0)$ \\
\hline $\mathrm{p}^{+t}$ & & 0.470 & & & 0.889 & \\
\hline \multicolumn{7}{|c|}{$\begin{array}{l}\text { 'Right colon: includes the cecum and ascending colon; transverse colon: includes the } \\
\text { hepatic and splenic flexures; left colon: includes the descending colon; sigmoid colon } \\
\text { and rectum. } \\
\text { "Kruskal-Wallis test; BBPS: Boston Bowel Preparation Scale } \\
\text { Data are presented as n (\%). }\end{array}$} \\
\hline
\end{tabular}

Colonic cleansing is one of the main factors affecting colonoscopy quality. Bowel cleansing technique for colonoscopy has undergone significant changes over the course of time.

The first methods employed in colonic cleansing involved diet restriction for a few days, oral cathartics and cathartic enema use (9). These methods led to fluid and electrolyte imbalances. With the discovery of more effective purgatives, the earlier traditional few-day clear fluid diet was gradually replaced by the better tolerated fiber-free diets $(10,11)$.

In 1980, Davis et al. (12) reported that they had developed a polyethylene glycol electrolyte lavage solution (PEG) with minimal fluid and electrolyte absorption and secretion. Although this solution was effective and safe, the necessity of high volume consumption, high salt content, and unpleasant odor due to its sodium sulphate component has led to modifications in the solution and development of low volume osmotic laxatives (13).

In 1990, Vanner et al. (14) developed a low volume sodium phosphate solution that was better tolerated. However, in the 2000s, side-effects associated with sodium phosphate like electrolyte impairments and renal toxicity restricted its use to high-risk groups such as children, the elderly, and those with diseases such as kidney failure and hypertension (15). 
Low volume osmotic laxatives containing magnesium have been reported to be insufficient when used alone but are effective when combined with other agents such as sodium picosulphate. These agents, which are well tolerated and effective as compared to PEG, unfortunately have the risks of causing dehydration, electrolyte changes and magnesium retention due to osmotic activity (16).

Sennosides are stimulating laxative-purgatives frequently employed in the treatment of constipation via increasing colonic motility, accelerating colonic transit time, and reducing fluid electrolyte secretion (17). They are frequently used in addition to PEG regimen, but have been shown to be as effective as PEG by themselves (3). However, the role of sennosides alone in colonic cleansing is controversial (2).

Sennoside $A+B$ calcium salt was used as a purgative in this study. We did not use PEG and NaP, which are known to perform better cleansing at standard doses, since the improving effect of the enema might have been masked. In Sloots et al. (5) study, the basis for our hypothesis, colonic transit time was significantly shorter in patients with constipation than in those without. With pre-purgative enema administration in our study, BBPS scores increased, although the difference was not statistically significant. Although not statistically significant, constipation was higher in female patients in terms of Rome criteria. Additionally, abdominal surgery history which is described as a separate risk factor for constipation was significantly higher in female patients. Both these factors might be the reason of statistically higher right colon BBPS scores. In other words, pre-purgative enema use improved right colon cleansing in patients with constipation. No significant difference was observed in terms of other parameters, such as tolerance, complications, length of procedure, or polyp detection.

\section{CONCLUSION}

Use of enemas before purgatives increases right colon cleansing in patients with tendency to constipation, such as female gender and a history of previous abdominal surgery. Further studies are needed to establish patient-specific colonoscopy preparation protocols.

Ethics Committee Approval: Ethics committee approval was received for this study from the ethics committee of Çanakkale Onsekiz Mart University Clinical Research Ethical Committee.

Informed Consent: Written informed consent was obtained from patients who participated in this study.

Peer-review: Externally peer-reviewed.

Author Contributions: Concept - M.Y., I.Y., M.B.; Design - M.Y., H.D., F.Ç.; Supervision - H.D., I.Y., M.Y.; Resource - M.Y., H.D., F.Ç.; Materials - M.Y., I.Y., H.D.; Data Collection and/or Processing - M.Y., I.Y., M.B.; Analysis and/or Interpretation - M.Y., F.Ç., H.T.; Literature Search - M.Y., I.Y., H.D.; Writing Manuscript - M.Y., F.Ç., H.D.; Critical Reviews - M.Y., M.B., I.Y.

Acknowledgements: The authors thank to Emine Sert and Müjgan Çatalçam.

Conflict of Interest: No conflict of interest was declared by the authors.

\section{REFERENCES}

1. Kao D, Lalor E, Sandha G, Fedorak RN, van der Knoop B, Doornweerd $\mathrm{S}$, et al. A randomized controlled trial of four precolonoscopy bowel cleansing regimens. Can J Gastroenterol 2011; 25: 657-662. [CrossRef]

2. Wexner SD, BeckDE, Baron TH, Fanelli RD, Hyman N, Shen B, et al. A consensus document on bowel preparation before colonoscopy: prepared by a task force from the American Society of Colon and Rectal Surgeons (ASCRS), the American Society for Gastrointestinal Endoscopy (ASGE), and the Society of American Gastrointestinal and Endoscopic Surgeons (SAGES). Gastrointest Endosc 2006; 63: 894-909. [CrossRef]

3. Radaelli F, Meucci G, Imperiali G, Spinzi G, Strocchi E, Terruzzi V, et al. High-dose senna compared with conventional PEG-ES lavage as bowel preparation for elective colonoscopy: a prospective, randomized, investigator-blinded trial. Am J Gastroenterol 2005; 100: 2674-2680. [CrossRef]

4. Lever EL, Walter MH, Condon SC, Balasubramaniam K, Chen YK, Mitchell RD, et al. Addition of enemas to oral lavage preparation for colonoscopy is not necessary. Gastrointest Endosc 1992; 38: 369-372. [CrossRef]

5. Sloots CE, Felt-Bersma RJ. Effect of bowel cleansing on colonic transit in constipation due to slow transit or evacuation disorder. Neurogastroenterol Motil 2002; 14: 55-61. [CrossRef]

6. Drossman DA, Dumitrascu DL. Rome III: New standard for functional gastrointestinal disorders. Journal of gastrointestinal and liver diseases: J Gastrointestin Liver Dis 2006; 15: 237-241.

7. Lai EJ, Calderwood AH, Doros G, Fix OK, Jacobson BC. The Boston bowel preparation scale: a valid and reliable instrument for colonoscopyoriented research. Gastrointest Endosc 2009; 69: 620-625. [CrossRef]

8. Ayaz S, Hisar F. The efficacy of education programme for preventing constipation in women. Int J Nurs Pract 2014; 20: 275-282. [CrossRef]

9. Beck DE, Harford FJ, DiPalma JA. Comparison of cleansing methods in preparation for colonic surgery. Dis Colon Rectum 1985; 28: 491-495. [CrossRef]

10. Soweid AM, Kobeissy AA, Jamali FR, El-Tarchichi M, Skoury A, AbdulBaki $\mathrm{H}$, et al. A randomized single-blind trial of standard diet versus fiber-free diet with polyethylene glycol electrolyte solution for colonoscopy preparation. Endoscopy 2010; 42: 633-638. [CrossRef]

11. Puckett J, Soop M. Optimizing colonoscopy preparation: the role of dosage, timing and diet. Curr Opin Clin Nutr Metab Care 2012; 15: 499-504. [CrossRef]

12. Davis GR, Santa Ana CA, Morawski SG, Fordtran JS. Development of a lavage solution associated with minimal water and electrolyte absorption or secretion. Gastroenterology 1980; 78: 991-995.

13. Corporaal S, Kleibeuker JH, Koornstra JJ. Low-volume PEG plus ascorbic acid versus high-volume PEG as bowel preparation for colonoscopy. Scand J Gastroenterol 2010; 45: 1380-1386. [CrossRef]

14. Vanner SJ, MacDonald PH, Paterson WG, Prentice RS, Da Costa LR, Beck IT. A randomized prospective trial comparing oral sodium phosphate with standard polyethylene glycol-based lavage solution (Golytely) in the preparation of patients for colonoscopy. Am J Gastroenterol 1990; 85: 422-427.

15. Rex DK, Vanner SJ. Colon cleansing before colonoscopy: does oral sodium phosphate solution still make sense? Can J Gastroenterol 2009; 23: 210-214. [CrossRef]

16. Adamcewicz M, Bearelly D, Porat G, Friedenberg FK. Mechanism of action and toxicities of purgatives used for colonoscopy preparation. Expert Opin Drug Metab Toxicol 2011; 7: 89-101. [CrossRef]

17. Kolts BE, Lyles WE, Achem SR, Burton L, Geller AJ, MacMath T. A comparison of the effectiveness and patient tolerance of oral sodium phosphate, castor oil, and standard electrolyte lavage for colonoscopy or sigmoidoscopy preparation. Am J Gastroenterol 1993; 88: 1218-1223. 